\title{
Positive Effect of HPA Lanolin versus Expressed Breastmilk on Painful and Damaged Nipples during Lactation
}

\author{
M. Abou-Dakn ${ }^{a}$ J.W. Fluhr ${ }^{\text {b, c }}$ M. Gensch ${ }^{a} \quad$ A. Wöckel ${ }^{d}$ \\ a Department of Obstetrics and Gynaecology, St. Joseph Hospital, Academic Hospital of the Charité, University \\ Medicine Berlin, ${ }^{b}$ Bioskin and ${ }^{\mathrm{C}}$ Department of Dermatology, Charité University Clinic, Berlin, and ${ }^{\mathrm{d}}$ Department of \\ Obstetrics and Gynaecology, University Hospital Ulm, Ulm, Germany
}

\section{Key Words}

Breastfeeding $\cdot$ Breastmilk $\cdot$ Highly purified anhydrous lanolin · Lactation • Nipple pain • Nipple trauma • Topical treatment

\begin{abstract}
Painful and/or damaged nipples associated with breastfeeding are common and represent a challenge for both the persons experiencing nipple pain and/or trauma and for those providing treatment. However, evidence-based data has been insufficient to demonstrably minimize these common reasons for failure to initiate or continue successful breastfeeding. The aim of this study was to evaluate the efficacy of specific-grade highly purified anhydrous (HPA) lanolin versus expressed breastmilk (EBM) for the treatment of painful and damaged nipples associated with breastfeeding in a prospective controlled clinical trial evaluating 84 lactating mothers. Nipple trauma and healing rates were rated by the Nipple Trauma Score. Nipple pain intensity was assessed on a visual analog scale. Outcome parameters were in favor of the HPA lanolin group, reaching statistical significance for healing rates, nipple trauma and nipple pain. In our study, we found HPA lanolin more effective than EBM, inducing faster healing of nipple trauma (absolute risk reduction of 0.43 ) and reducing nipple pain (absolute risk reduction of 0.61 on day
\end{abstract}

3). We concluded that HPA lanolin, combined with breastfeeding education, was more effective than EBM, combined with breastfeeding education, in reducing nipple pain and promoting healing of nipple trauma.

Copyright $\odot 2010$ S. Karger AG, Basel

\section{Introduction}

Nipple pain and/or trauma associated with breastfeeding are common, with incidences varying between 34 and $96 \%$ [1-4], and are cited as one of the main reasons for early cessation of breastfeeding in the early postpartum period, while later on, low milk supply is often seen as a reason to stop breastfeeding. Treating sore and/or traumatized nipples quickly and effectively is therefore an important factor in establishing successful breastfeeding by maintaining a pleasurable breastfeeding relationship between mother and infant and preventing complications such as mastitis [1, 3, 4] or breast abscesses [5].

A variety of interventions have been used to either treat or prevent nipple pain and/or trauma associated with breastfeeding. These include topical creams, solutions or sprays, time-restricted breastfeeding, exposure of the nipples to dry heat or ultraviolet light and air-drying, hardening of the nipple skin, and pre- or postnatal breastfeed-

\section{KARGER}

Fax +41613061234 E-Mail karger@karger.ch www.karger.com
(C) 2010 S. Karger AG, Basel

1660-5527/11/0241-0027\$38.00/0

Accessible online at:

www.karger.com/spp
Joachim W. Fluhr, MD

Bioskin

Bergmannstrasse 5

DE-10961 Berlin (Germany)

Tel. +49 302804 3950, E-Mail joachim.fluhr@gmx.net 
ing education $[1,3,6-8]$. However, there is little evidence from prospective trials regarding the use of topical agents [9-13]. Despite a lack of evidence-based research on its efficacy, expressed breastmilk (EBM) continues to be widely recommended for the prevention and treatment of sore and/or damaged nipples. While EBM has immune globulin content and heals damaged intestines internally, two sources have suggested that it also could promote healing of injured skin [1]. Lanolin, an organic ester derived from sheep fleece after shearing, creates an air-permeable temporary barrier and promotes moist wound healing when applied to injured skin. Lanolin is proven to have antiinflammatory, antimicrobial, skin-protecting and barrier repair properties $[14,15]$. There are many grades of lanolin with varying degrees of purity and safety available. The grades range from crude industrial grades to an ultrapurified medical grade, highly purified anhydrous (HPA) lanolin, the specific type of lanolin we used in this study. HPA lanolin is purified by a special proprietary process in which impurities and allergenic components are removed thereby rendering it free of odor, taste, bleaches and preservatives. This is important as it is applied to the mother's nipples and may be ingested by the infant and because having to remove an impure lanolin product or other topical ointment can exacerbate existing soreness or injury and thereby lead to early cessation of breastfeeding [16]. Allergies to lanolin amongst the general population are very rare (1.46-8.75 cases per million) [17]. HPA lanolin is ultrarefined to remove free lanolin alcohols to a level lower than $1.5 \%$ and to lower detergent residues to a negligible level and is, thereby, rendered hypoallergenic. Furthermore the purification process removes any pesticide residues to below $1 \mathrm{ppm}$. The irritation and sensitization potential of topically applied products is an ongoing matter of debate [18, 19].

The aim of this study was to prospectively compare the use of EBM topical treatments of sore nipples during breastfeeding with HPA lanolin against EBM.

\section{Material and Methods}

\section{Study Design}

A prospective controlled clinical trial was carried out to evaluate the efficacy of HPA lanolin compared to EBM. The outcome parameters were healed nipples, nipple pain, cracked nipples, breastfeeding duration and the incidence of related breastfeeding complications. The allocation of women into the intervention group or the control (EBM) group was done depending on whether her pain was reported for the first time on a day with an even or odd date. Therefore, this study does not meet the full criteria for a randomized trial. Recruiting was done by a study nurse, and the visits, data collection and evaluation were carried out by physicians in order to reduce potential bias of the allocation of women into the groups. The study was performed in accordance with the Declaration of Helsinki published in 1997. The local Institutional Review Board (ethics commission) approved the study. An information sheet about the study was provided, and written informed consent was obtained from all participants. The study design and the allocation are represented in figure 1.

\section{Recruitment}

Recruitment and treatment were carried out at the delivery wards of two hospitals certified by the Baby-Friendly Hospital Initiative (BFHI) in Berlin, Germany - Humboldt Hospital and St. Joseph Hospital - between September 2005 and February 2007. The study sample consisted of healthy breastfeeding women older than 18 years who spoke German, had a low-risk pregnancy, delivered a singleton child of greater than 37 weeks of gestation with no neonatal complications, were receiving mother-infant combined care and developed nipple pain while breastfeeding within $72 \mathrm{~h}$ after delivery. All participants visited a birth preparation class in the second trimester and were given identical information related to breastfeeding including the potential for problems and complications that could be experienced during the lactation period. Exclusion criteria were anomalies in breast and/or nipple anatomy, chronic illness and the presence of other persistent pain-related conditions. No statistical differences were detectable between the two groups regarding ethnicity, education level, smoking habits, mode of delivery, prenatal classes attended or microbiological colonization.

\section{Data Collection}

Eligible women in the delivery ward were approached daily by the researcher and asked to rate the nipple pain they experienced while breastfeeding by marking their pain intensity level on a visual analog scale (VAS) form given to them ranging from 0 (no pain) to 10 (most intense pain imaginable). All types of pain variations like stabbing pain, acute pain and/or burning pain while breastfeeding or between feeds were classified using the same VAS. Women who developed nipple pain ratings of 2 or more on the VAS within $72 \mathrm{~h}$ after delivery in one or both breasts were invited to take part in the trial and were given written instructions for study participation. The VAS is accepted as a valid and reliable measure for a variety of subjective phenomena and has been previously used to measure nipple pain [20,21]. Pain was assessed on enrollment (baseline) and days 3, 7 and 14 after enrollment.

Prior to enrollment, mothers were given no advice or options regarding the use of EBM or lanolin. To avoid any switching between the groups, both treatments were described as relatively equal when training the recruiting nurses. At enrollment, all women received specific instructions regarding their allocated treatment modality. Women of both groups were instructed to wash their hands with water and soap prior to breastfeeding and using either treatment. Women using EBM were instructed to express and to massage a few drops of EBM on the nipples and areola after each feed, which were then allowed to air-dry. Women using HPA lanolin were instructed to pat the nipples dry after each feeding and apply a pea-sized amount of HPA lanolin (approximately $0.25 \mathrm{~g}$ ) to the nipple and areola and to keep this area covered with HPA lanolin at all times, including between feedings 
Fig. 1. Flow chart of design and patient allocation.

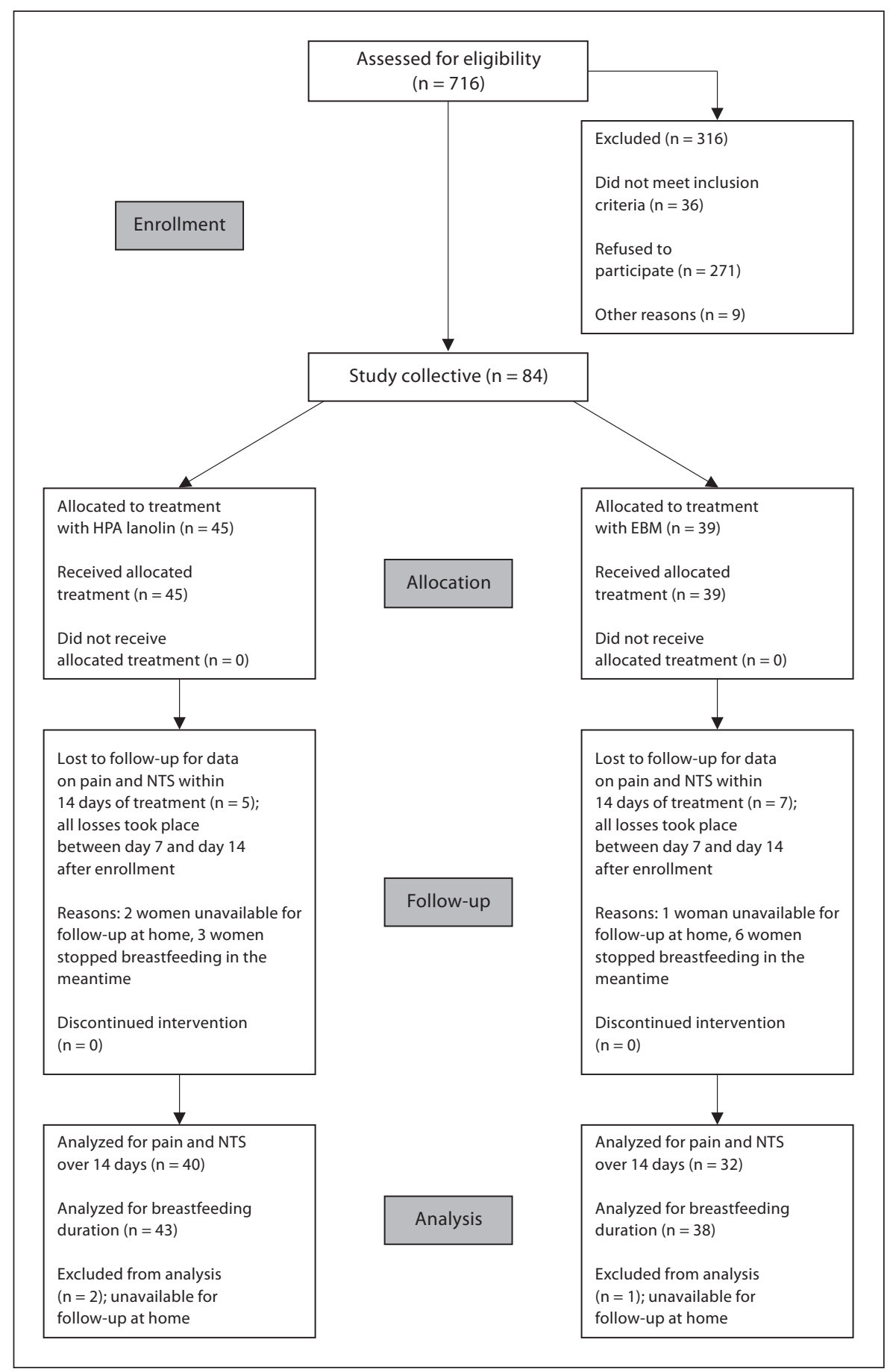

in order to create a moist healing environment. Mothers were instructed to not wash off the HPA lanolin before the next feeding. At enrollment, a microbiological swab of both mammilla regions was taken to identify possible baseline differences of physiological cutaneous bacterial colonization between the two groups. Women were instructed to avoid applying devices or any other topical treatment for nipple pain and nipple trauma.

HPA Lanolin to Treat Painful and Damaged Nipples during Lactation
After enrollment (baseline), patients were seen by a physician on days 3, 7 and 14 after the commencement of nipple pain. For evaluation of fatigue we used a scale from 0 to 10 indicating higher fatigue value with increasing scale figure $(0$ means 'no fatigue at all', 10 means 'most fatigue imaginable') [22]. The standardized 20 -item Perceived Stress Questionnaire was used to rate maternal stress $[23,24]$. Mastitis was differentiated from milk stasis by oc- 
Successful
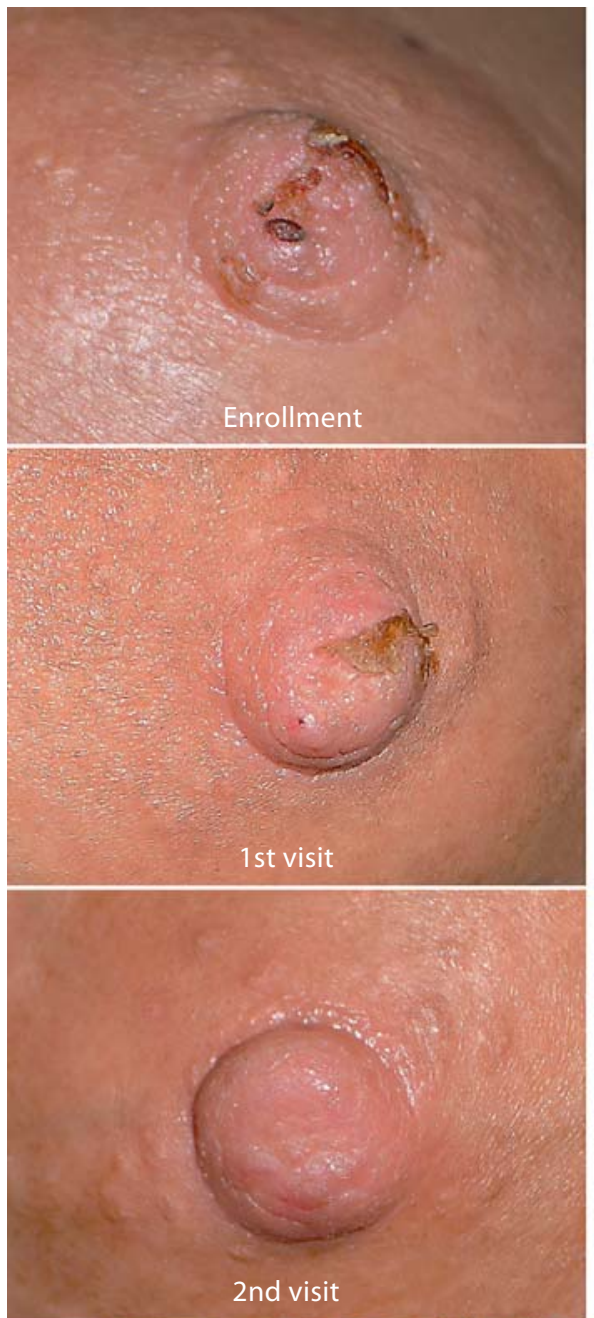

2nd visit

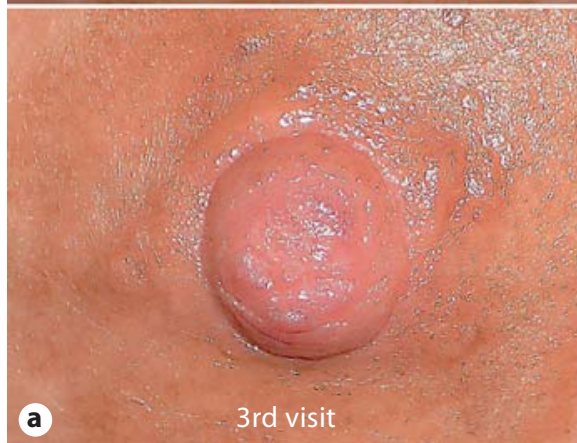

\section{a}

Nonsuccessful
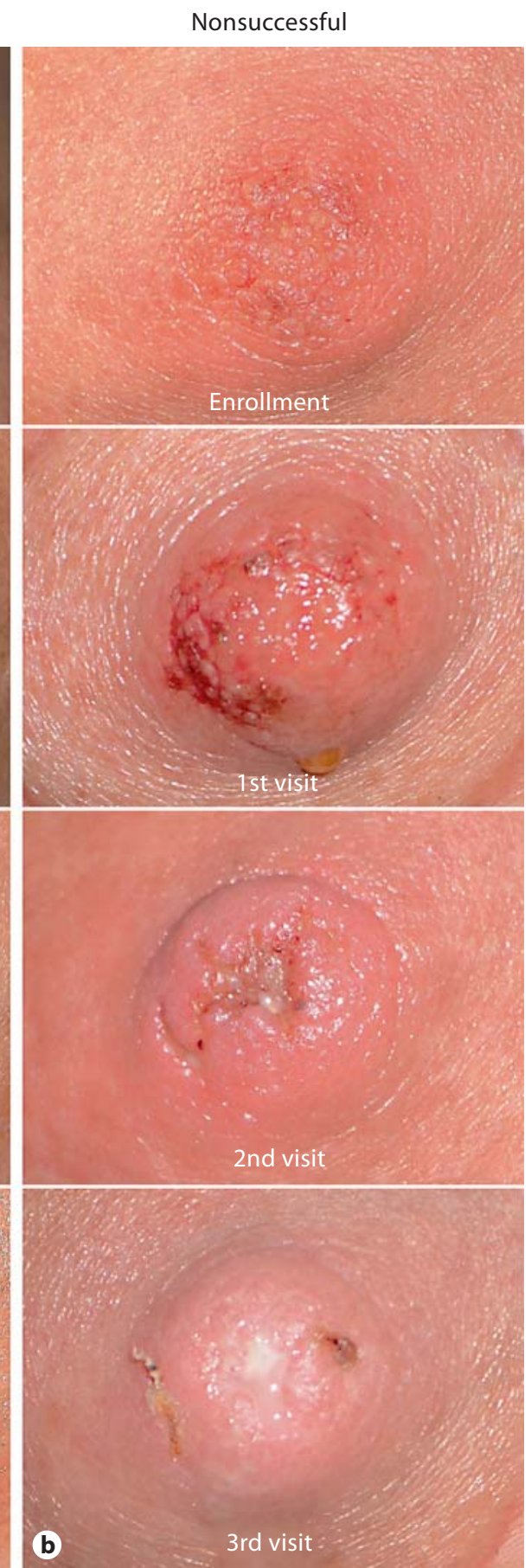

(b) 3rd visit
Fig. 2. Typical series of successful wound healing and nonsuccessful treatment. currence symptoms like massive hyperthermia, redness, fever $>38^{\circ} \mathrm{C}$, profound systemic illness after conservative measures like massage and emptying the breast.

During each visit, standardized photographs were taken of both nipples to later identify and rate nipple trauma (baseline, day 3 , day 7 and day 14; fig. 2 shows an example for different degrees and rating of nipple trauma) after commencement of nipple pain.
Nipple trauma is defined as a macroscopic traceable cutaneous lesion in the area of the mammilla and areola, which may accumulate in the form of fissures, eroded skin and ulcerations [25]. The Nipple Trauma Score (NTS) was used to identify and score nipple trauma and to evaluate healing rates of both breasts separately in this study. The NTS is based on wound depth and the extent of tissue damage (table 1). Testing of NTS revealed a high 
Table 1. Description of the NTS

0 No microscopically visible skin changes

1 Erythema or edema or combination of both

2 Superficial damage with or without scab formation of less than $25 \%$ of the nipple surface

3 Superficial damage with or without scab formation of more than $25 \%$ of the nipple surface

4 Partial-thickness wound with or without scab formation of less than $25 \%$ of the nipple surface

5 Partial-thickness wound with or without scab formation of more than $25 \%$ of the nipple surface

interobserver reliability of 0.88 (Goodman's gamma). A nipple was considered to be 'healed' if the corresponding trauma score rating was either 0 or 1 , the important factor being that the nipple surface was intact. If there was a difference of opinion on the photographs between the two observers, they discussed their evaluations in order to reach consensus about the rating of individual nipples. Follow-up telephone calls were made by a physician at 4 , 12 and 32 weeks after delivery to confirm breastfeeding status and detect possible complications related to weaning.

\section{Data Analysis}

Previous breastfeeding studies within the same population and geographic region where this study was performed showed a $20 \%$ dropout rate due to a prevalence of breastfeeding-dependent breast problems (pain $[20,26]$, nipple trauma [25]). Assuming a $20 \%$ loss of participation within the study group, we calculated that we would require 80 breastfeeding mothers to detect a statistically significant difference of $15 \%(1-\alpha=95 \%, 1-\beta=80 \%)$ in the ratings of nipple trauma and pain intensity [27]. All data were analyzed as intention to treat. Data were analyzed using SPSS version 15.0 for Windows (SPSS Inc., Chicago, Ill., USA): nonparametric Mann-Whitney test, parametric t test (after testing for normal distribution) and $\chi^{2}$ test. $\mathrm{p}<0.05$ was considered statistically significant. The correlation between wound score and reports on pain were calculated with $t$, paired $t$ and $\chi^{2}$ tests.

\section{Results}

A total of 84 women were enrolled in the trial, with 45 participants allocated to the HPA lanolin group and 39 to the EBM group. This led to the number of $n=90$ included breasts in the HPA lanolin group and to $n=78$ in the EBM group. Three women were unavailable for follow-up at home though data from their visits at the hospital and telephone interviews were included in the study. Comparison of the two groups with respect to demographic indicators collected at the time of enrollment did not

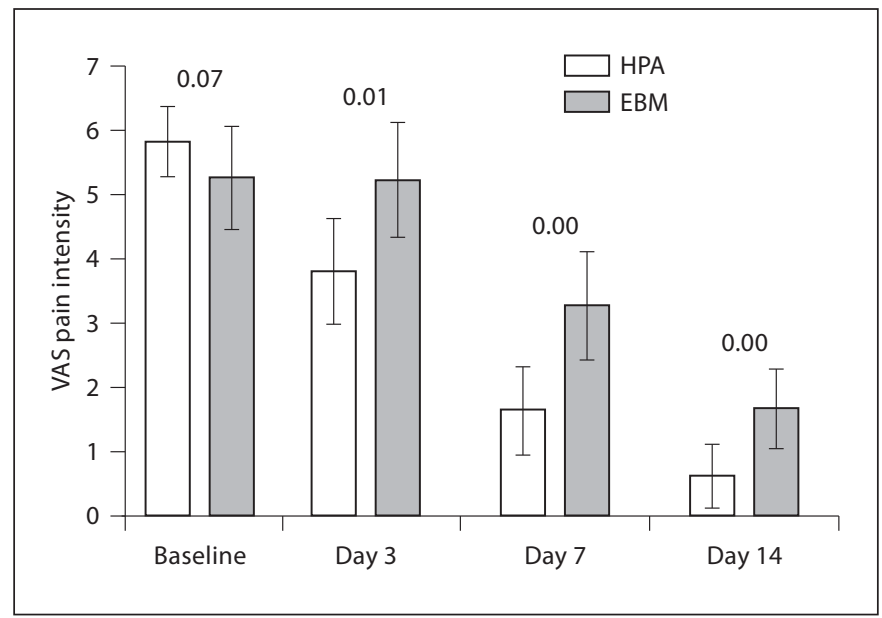

Fig. 3. Pain intensity while breastfeeding assessed via VAS ranging from 0 to 10 compared by Mann-Whitney test: no significant difference was noted at baseline. On all days, a significantly lower pain level was detectable in the HPA group; mean values $\pm 95 \%$ confidence intervals.

reveal any statistically significant difference between the study groups (data not shown). Results from the nipple swabs taken at enrollment yielded no statistically significant results between the two groups, only physiological skin flora was grown (data not shown). Comparison of the mother's handedness revealed no statistically significant differences.

Pain, nipple trauma, fatigue and maternal stress were the measured outcomes due to findings that show that fatigue and maternal stress can be disruptive for the breastfeeding relationship between mother and child $[28,29]$.

\section{Pain}

On the third day after enrollment, we found significant differences in pain score between the two treatment groups (fig. 3). The group using HPA lanolin showed a faster decrease in nipple pain intensity during feedings. In this group, nipple pain began to decrease with the commencement of treatment, while in the group using EBM, pain intensity initially increased and peaked on the third day postpartum. The difference in self-reported pain intensity between the two groups reached statistical significance on all days except the day of enrollment after the first use of either EBM or HPA lanolin, with lower pain scores being reported by women using HPA lanolin. Individual breasts showed clear differences when looking at changes in pain in the detailed analysis (improvement, 
Table 2. Changes of pain of individual breasts (numbers, with percentages in parentheses) relating to day of enrollment ( $\chi^{2}$ test): HPA-lanolin-treated patients experienced significantly less pain at all time points

\begin{tabular}{llll}
\hline & $\begin{array}{l}\text { Development until } \\
\text { 1st visit (3rd day } \\
\text { after enrollment) }\end{array}$ & $\begin{array}{l}\text { Development until } \\
\text { 2nd visit (7th day } \\
\text { after enrollment) }\end{array}$ & $\begin{array}{l}\text { Development until } \\
\text { 3rd visit (14th day } \\
\text { after enrollment) }\end{array}$ \\
\hline $\begin{array}{l}\text { Treatment with HPA lanolin } \\
\text { Improvement }\end{array}$ & $43(65)$ & $66(87)$ & $69(89)$ \\
$\quad \begin{array}{l}\text { No change } \\
\text { Deterioration }\end{array}$ & $14(21)$ & $5(6)$ & $0(0)$ \\
$\begin{array}{l}\text { Treatment with EBM } \\
\text { Improvement } \\
\text { No change }\end{array}$ & $9(14)$ & $5(6)$ & $1(1)$ \\
$\quad$ Deterioration & $18(30)$ & $47(71)$ & $51(88)$ \\
$\chi^{2}$ test & $13(22)$ & $5(8)$ & $2(3)$ \\
\hline
\end{tabular}

deterioration, no change) between both groups (table 2): in the period from enrollment until the first follow-up visit, there was a pain reduction in $65 \%(n=43)$ of the examined breasts in the group using HPA lanolin, whereas in the group using EBM, this number was only $30 \%$ ( $n=$ 18). This corresponds to an absolute risk reduction of 0.61 regarding pain reduction within the first 3 days after commencement of treatment with HPA lanolin. In the group using EBM, pain initially worsened in the majority of the examined breasts $(48 \% ; n=29)$, an effect that was not observed in the HPA lanolin group, where pain worsened in only $14 \%(n=9 ; p<0.001)$. From enrollment until the second visit, the percentage of nipple pain improvement in the HPA lanolin group was $87 \%(n=66)$, i.e. significantly higher than in the EBM group, where $71 \%(\mathrm{n}=$ 47) had experienced improvement until day $7(p=0.034)$. By the third visit, there was also a statistically significant difference in pain between the two study groups, with $89 \%(n=69)$ of nipples treated with HPA lanolin having experienced nipple pain improvement compared to $88 \%$ $(\mathrm{n}=51)$ in the group using EBM $(\mathrm{p}=0.043)$.

\section{Nipple Trauma}

Macroscopically visible changes of the mammilla of at least one breast (NTS $\geq 1$ ) were seen in $90 \%$ of participants at the beginning of the study even though the enrollment criterion was breastfeeding-related pain only (VAS $\geq 2$ ). The highest incidence of wound development of the mammilla due to breastfeeding was noticed on the third day postpartum. A statistically significant difference in physician-rated nipple trauma could be observed on all days except the day of enrollment, with trauma being severer in the group using EBM (fig. 4).

\section{Healing Rates}

In the group using HPA lanolin, there were significantly greater numbers of healed nipples at the first ( $\mathrm{n}=28 \mathrm{vs}$. $\mathrm{n}=11)$ and second visits $(\mathrm{n}=52$ vs. $\mathrm{n}=27)$ after initiating the topical treatment. At the third visit, the number of healed nipples in the HPA lanolin group was greater than in the EBM group ( $\mathrm{n}=52$ vs. $\mathrm{n}=38$ ); however, this difference did not reach statistical significance (fig. 5). There was a statistically significant increase in new skin defects from the day of enrollment until the first visit in the group using EBM (43\%; $n=24)$, which was not observed in the group using HPA lanolin, where this number was 39\% $(\mathrm{n}=22 ; \mathrm{p}=0.107)$. This corresponds to an absolute risk reduction of 0.43 regarding prevention of new skin defects within the first 3 days of treatment with HPA lanolin.

\section{Breastfeeding Duration, Complications and Maternal Stress}

There was a significant difference in breastfeeding cessation rates during the first 14 days of treatment. Of the 39 mothers using EBM, 6 stopped breastfeeding (15\%) whereas only 3 of the 45 mothers using HPA lanolin stopped breastfeeding (7\%) during that same time period. Follow-up on breastfeeding status revealed no statistically significant difference in breastfeeding duration between the two groups at 32 weeks postpartum; a total of $69 \%$ of all women continued to breastfeed their children. There was, however, a difference in the pattern of complications between the two groups: in the group using EBM, 8 women suffered from breast engorgement and 3 developed mastitis; in the HPA lanolin group, only 4 women developed breast engorgement and there were no cases of mastitis. There were no noted side effects due to the treat- 


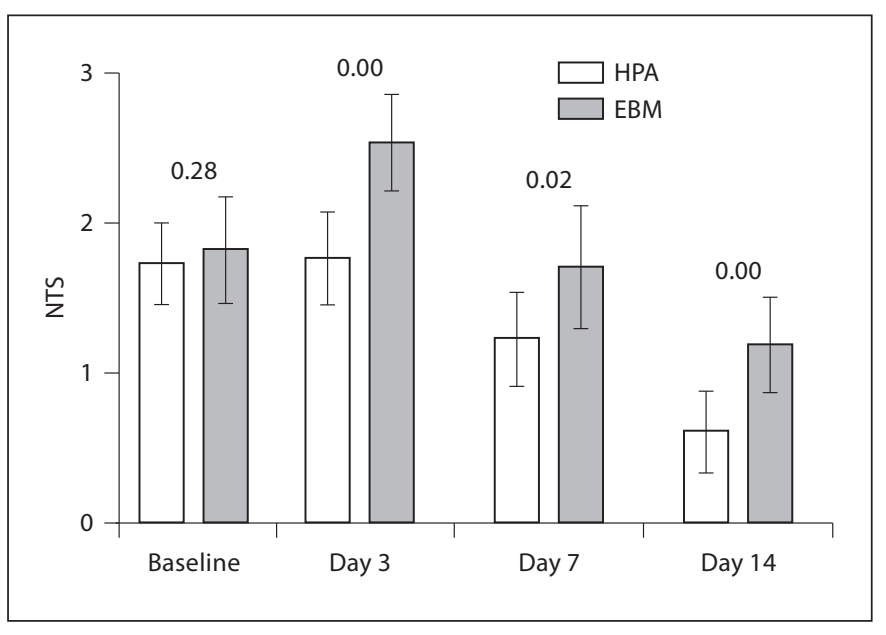

Fig. 4. Nipple trauma assessed with the NTS ranging from 0 to 5 (table 1) compared by the Mann-Whitney test: no significant difference was noted at baseline. On all days, a significantly lower NTS level was detectable in the HPA group; mean values $\pm 95 \%$ confidence intervals.

ments in either group. None of the participants switched groups. There was no significant difference between the groups concerning fatigue or stress measured by the Perceived Stress Questionnaire at any time (data not shown).

\section{Discussion}

This intervention study demonstrates the beneficial effects of HPA lanolin in the treatment of sore and/or damaged nipples associated with breastfeeding as compared to the topical application of EBM. As in other studies, all data were collected for each breast separately $[1$, 11]. The appropriate use of HPA lanolin results in a significant reduction of pain associated with breastfeeding and significantly higher healing rates of nipple trauma within 14 days of topical treatment. Benefits are most pronounced within the first 3 days of treatment. Furthermore, the group using EBM developed 3 cases of mastitis while the HPA lanolin patients did not develop any cases of mastitis. At 32 weeks postpartum, there was no statistically significant difference between the two groups regarding breastfeeding duration, fatigue and maternal stress. It should be noted and emphasized that there was no correlation between pain score and wound score in either group, which should be taken into consideration when advising breastfeeding mothers with painful nipples.

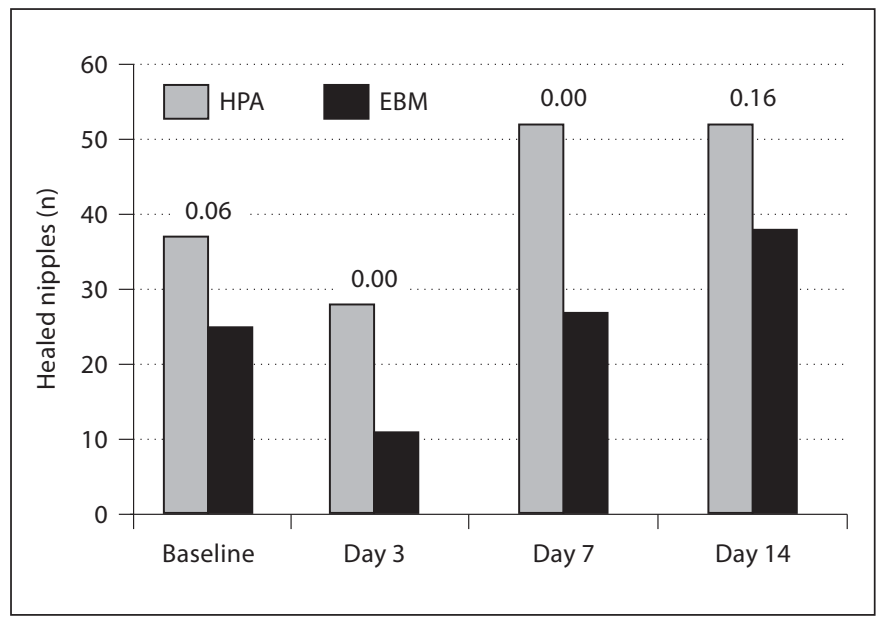

Fig. 5. Number of healed nipples compared by $\chi^{2}$ test: no difference was noted at baseline and day 14, but a significantly higher healing rate was observed in the HPA lanolin group on days 3 and 7.

In recent years topical treatment for anti-irritant and wound healing has been studied [30, 31]. A comprehensive review of the literature reveals that nipple pain comprises a substantial number of preventable breastfeeding problems. A mother who attempts to forestall the pain may cause the suckling stimulation to be limited, thus curtailing the secretion of milk $[1,20]$. The pain may also inhibit the let-down reflex so that the milk already in the breast does not flow out to the baby [32]. With decreased milk flow, the negative pressure exerted by the baby's suction on the nipple increases and may lead to further nipple damage [1]. Early pain experienced by a breastfeeding mother can also have a negative psychological impact [33]. It is imperative then that the mother receives symptomatic relief, which will likely help her to relax while she works on the underlying cause of the trauma, which may well be a matter of correctly positioning the baby at the breast or fixing a faulty latch by the baby attaching to the breast. When sore nipples are not treated adequately, tissue breakdown can progress rapidly and, in some cases, nipple injury can be extensive [34].

Nipple tissue breakdown can give way to complications and breast infections such as mastitis, as broken skin is likely to provide a portal for entry of bacteria. Traumatized nipples can readily become infected with bacteria or yeast, the presence of which can delay healing even when positioning and latch-on are corrected. A 2002 study found that the presence of cracks and nipple sores was associated with an almost sixfold increase in 
mastitis rates for women with no history of mastitis, and a threefold increase in women with such a history [35].

With the increased use of moist wound healing in general wound treatment, the same theories are being applied to the management of sore and/or damaged nipples. In moist wound healing, a moist environment is created at the injury site which is favorable for reepithelization. HPA lanolin has been shown to have anti-inflammatory and barrier repair properties $[14,15]$. By placing a temporary moisture barrier over the injured skin site using HPA lanolin, the proliferation and migration of epithelial cells across the surface of the wound are enhanced during healing. Moist wound healing eliminates crust formation and has been shown to encourage reepithelization up to $50 \%$ faster.

Previous studies have shown that nipple pain related to breastfeeding peaks on the third day after birth $[1,36]$. Various studies have identified nipple pain and/or trauma as the main reason for early cessation of breastfeeding in this period [37]. Based on the outcomes of this trial, while the underlying cause of nipple pain is being identified and corrected, a topical treatment other than EBM appears to be beneficial especially in the critical period of initiation of breastfeeding.

The beneficial effects of lanolin in the prevention or treatment of sore or damaged nipples have been demonstrated before $[1,8,38]$. However, in these trials lanolin has always been compared to or combined with other interventions, and the purity, quality and form of the lanolin and the frequency of application have varied. HPA is a type of highly purified, medical-grade lanolin that has had allergenic components and environmental impurities removed by a proprietary process rendering it safe for use by breastfeeding mothers and infants.

Even though this study has been designed to meet the methodological criteria of a prospective controlled trial (e.g. intention to treat, larger sample sizes than in previous studies, inclusion of risk reduction and inclusion of adverse effects), a certain methodological bias could not be entirely avoided, because the allocation to the intervention or control group was done by date and not by a randomization. In addition, double blinding is difficult to obtain in studies concerning breastfeeding problems. Therefore, a single-blinded design was used with collection of data and data analysis being performed by different investigators. The evaluation of the photodocumentation of individual nipples was conducted by independent reviewers. The doctors examining the photos could not allocate them in pairs in order to ensure an independent observation of each nipple of the study participants.
Due to frequent visits and phone calls as part of the study design, the study participants received more intensive breastfeeding instruction than nonparticipants. As a result, incidences of breastfeeding problems might be lower in both groups than breastfeeding problems among breastfeeding women who give birth in non-BFHI hospitals where breastfeeding may not be stressed as important as in BFHI hospitals. In hospitals that are not certified as Baby Friendly, women may get standard or substandard breastfeeding attention and accommodations. Breastfeeding education has previously been shown as beneficial for avoidance of breastfeeding problems [12]. For example, the rate of mastitis in the control/EBM group was only $14 \%$ after a period of 32 weeks in a high-risk group, whereas in other studies done in non-BFHI hospitals, this rate has been reported to be as high as 30\% [39].

In all interviews, we asked about the application of each treatment. All participants stated correct and full compliance with the therapy. Not a single case was reported of a changeover to the other treatment. However, it is assumed that some responses of women might not be correct in all cases because they wanted to meet expectations. Therefore, the benefits of HPA lanolin concerning breastfeeding duration might be masked and should be confirmed in further research.

In conclusion, our data shows a significantly better outcome for patients treated with topical HPA lanolin as compared to EBM. This benefit reached statistical significance for healing rates, nipple trauma and nipple pain. In our study topical treatment with HPA lanolin is more effective than EBM for faster healing of nipple trauma and reducing nipple pain. We conclude from our study that the current recommendation for any topical treatment of sore nipples during breastfeeding should be revised in favor of HPA lanolin. If pathology of nipple pain and cracked nipples worsens, a reevaluation should be carried out independently to exclude pathophysiological factors leading to advancement of milk stasis or mastitis puerperalis.

\section{Acknowledgements}

The authors are grateful to the staff at the Humboldt Hospital and St. Joseph Hospital for their help, and to the mothers who participated in the study. We would also like to thank the former director of the Humboldt Clinic, Prof. J.R. Strecker, who enabled and fully supported the study scientifically and logistically. Funding was provided by a grant from St. Joseph Hospital, Berlin, Germany. 


\section{References}

1 Akkuzu G, Taskin L: Impacts of breast-care techniques on prevention of possible postpartum nipple problems. Prof Care Mother Child 2000;10:38-41.

-2 Gartner LM, Morton J, Lawrence RA, Naylor AJ, O'Hare D, Schanler RJ, Eidelman AI: Breastfeeding and the use of human milk. Pediatrics 2005; 115:496-506.

-3 Lavergne NA: Does application of tea bags to sore nipples while breastfeeding provide effective relief? J Obstet Gynecol Neonatal Nurs 1997;26:53-58.

4 Tait P: Nipple pain in breastfeeding women: causes, treatment, and prevention strategies. J Midwifery Womens Health 2000;45:212215.

-5 Eryilmaz R, Sahin M, Hakan Tekelioglu M, Daldal E: Management of lactational breast abscesses. Breast 2005;14:375-379.

6 Herd B, Feeney JG: Two aerosol sprays in nipple trauma. Practitioner 1986;230:31-38.

7 Lochner JE, Livingston CJ, Judkins DZ: Clinical inquiries: which interventions are best for alleviating nipple pain in nursing mothers? J Fam Pract 2009;58:612a-612c.

$\checkmark 8$ Pugh LC, Buchko BL, Bishop BA, Cochran JF, Smith LR, Lerew DJ: A comparison of topical agents to relieve nipple pain and enhance breastfeeding. Birth 1996;23:88-93.

-9 Amir LH, Lumley J, Garland SM: A failed RCT to determine if antibiotics prevent mastitis: cracked nipples colonized with Staphylococcus aureus - a randomized treatment trial (ISRCTN65289389). BMC Pregnancy Childbirth 2004;4:19.

10 Livingstone V, Stringer LJ: The treatment of Staphyloccocus aureus infected sore nipples: a randomized comparative study. J Hum Lact 1999;15:241-246.

11 Melli MS, Rashidi MR, Nokhoodchi A, Tagavi S, Farzadi L, Sadaghat K, Tahmasebi Z, Sheshvan MK: A randomized trial of peppermint gel, lanolin ointment, and placebo gel to prevent nipple crack in primiparous breastfeeding women. Med Sci Monit 2007; 13:CR406-CR411.

$\checkmark 12$ Morland-Schultz K, Hill PD: Prevention of and therapies for nipple pain: a systematic review. J Obstet Gynecol Neonatal Nurs 2005;34:428-437.

-13 Sayyah Melli M, Rashidi MR, Delazar A, Madarek E, Kargar Maher MH, Ghasemzadeh A, Sadaghat K, Tahmasebi Z: Effect of peppermint water on prevention of nipple cracks in lactating primiparous women: a randomized controlled trial. Int Breastfeed J 2007;2:7.

14 Hoppe U (ed): The Lanolin Book. Hamburg, Beiersdorf, 1999.
15 Elias PM, Man M-Q, Thornfeldt CR, Feingold KR: The epidermal permeability barrier: effects of physiologic and non-physiologic lipids; in Hoppe U (ed): The Lanolin Book. Hamburg, Beiersdorf, 1999, vol 3, pp 253 278.

16 Rissmann R, Oudshoorn MH, Kocks E, Hennink WE, Ponec M, Bouwstra JA: Lanolinderived lipid mixtures mimic closely the lipid composition and organization of vernix caseosa lipids. Biochim Biophys Acta 2008; 1778:2350-2360.

17 Clark EW, Blondeel A, Cronin E, Oleffe JA, Wilkinson DS: Lanolin of reduced sensitizing potential. Contact Dermatitis 1981;7:8083 .

18 Basketter D, Darlenski R, Fluhr JW: Skin irritation and sensitization: mechanisms and new approaches for risk assessment. Skin Pharmacol Physiol 2008;21:191-202.

19 Fluhr JW, Darlenski R, Angelova-Fischer I, Tsankov N, Basketter D: Skin irritation and sensitization: mechanisms and new approaches for risk assessment. 1. Skin irritation. Skin Pharmacol Physiol 2008;21:124135.

20 Duffy EP, Percival P, Kershaw E: Positive effects of an antenatal group teaching session on postnatal nipple pain, nipple trauma and breast feeding rates. Midwifery 1997;13:189_ 196.

21 Buchko BL, Pugh LC, Bishop BA, Cochran JF, Smith LR, Lerew DJ: Comfort measures in breastfeeding, primiparous women. J Obstet Gynecol Neonatal Nurs 1994;23:46-52.

22 Sherman SA, Eisen S, Burwinkle TM, Varni JW: The PedsQL present functioning visual analogue scales: preliminary reliability and validity. Health Qual Life Outcomes 2006;4: 75 .

23 Levenstein S, Prantera C, Varvo V, Scribano ML, Berto E, Luzi C, Andreoli A: Development of the Perceived Stress Questionnaire: a new tool for psychosomatic research. J Psychosom Res 1993;37:19-32.

24 Fliege H, Rose M, Arck P, Walter OB, Kocalevent RD, Weber C, Klapp BF: The Perceived Stress Questionnaire (PSQ) reconsidered: validation and reference values from different clinical and healthy adult samples. Psychosom Med 2005;67:78-88.

25 Wockel A, Schumann M, Abou-Dakn M Development of a wound score for the evaluation of cracked nipples during lactation. Arch Gyn Obst 2004;17(suppl 1):270.

- 26 Herr K, Spratt KF, Garand L, Li L: Evaluation of the Iowa Pain Thermometer and other selected pain intensity scales in younger and older adult cohorts using controlled clinical pain: a preliminary study. Pain Med 2007;8: 585-600.

27 Armitage P: Theory and practice in medical statistics. Stat Med 2001;20:2537-2548.
28 Groer M, Davis M, Casey K, Short B, Smith K, Groer S: Neuroendocrine and immune relationships in postpartum fatigue. MCN Am J Matern Child Nurs 2005;30:133-138.

29 Groer MW, Davis MW: Cytokines, infections, stress, and dysphoric moods in breastfeeders and formula feeders. J Obstet Gynecol Neonatal Nurs 2006;35:599-607.

-30 Daeschlein G, Assadian O, Bruck JC, Meinl C, Kramer A, Koch S: Feasibility and clinical applicability of polihexanide for treatment of second-degree burn wounds. Skin Pharmacol Physiol 2007;20:292-296.

31 Hughes-Formella B, Wunderlich O, Williams R: Anti-inflammatory and skin-hydrating properties of a dietary supplement and topical formulations containing oligomeric proanthocyanidins. Skin Pharmacol Physiol 2007;20:43-49.

- 32 Wockel A, Abou-Dakn M, Beggel A, Arck P: Inflammatory breast diseases during lactation: health effects on the newborn - a literature review. Mediators Inflamm 2008;2008: 298760.

-33 Amir LH, Dennerstein L, Garland SM, Fisher J, Farish SJ: Psychological aspects of nipple pain in lactating women. J Psychosom Obstet Gynaecol 1996;17:53-58.

34 Abou-Dakn M, Richardt A, Schaefer-Graf U, Wöckel A: Inflammatory breast diseases during lactation: milk stasis, puerperal mastitis, abscesses of the breast, and malignant tumors - current and evidence-based strategies for diagnosis and therapy. Breast Care 2010;5:33-37.

35 Foxman B, D’Arcy H, Gillespie B, Bobo JK, Schwartz K: Lactation mastitis: Occurrence and medical management among 946 breastfeeding women in the United States. Am J Epidemiol 2002;155:103-114.

36 Centuori S, Burmaz T, Ronfani L, Fragiacomo M, Quintero S, Pavan C, Davanzo R, Cattaneo A: Nipple care, sore nipples, and breastfeeding: a randomized trial. J Hum Lact 1999;15:125-130.

37 Waldenstrom U, Aarts C: Duration of breastfeeding and breastfeeding problems in relation to length of postpartum stay: a longitudinal cohort study of a national Swedish sample. Acta Paediatr 2004;93:669-676.

38 Dodd V, Chalmers C: Comparing the use of hydrogel dressings to lanolin ointment with lactating mothers. J Obstet Gynecol Neonatal Nurs 2003;32:486-494.

39 Wockel A, Beggel A, Gensch M, Abou-Dakn M: Psychological stress and puerperal mastitis: possible pathophysical mechanisms. Curr Womens Health Rev 2007;3:123-127. 